Abanico Veterinario. Enero-Diciembre 2020; 10:1-17. http://dx.doi.org/10.21929/abavet2020.24 Artículo Original. Recibido: 20/11/2019. Aceptado: 30/11/2020. Publicado: 14/12/2020. Clave: 2019-44.

\title{
Efecto de la suplementación con metionina de zinc en el desempeño productivo y morfología del epitelio intestinal en cerdos criados en ambiente caluroso o fresco
}

\author{
Zinc methionine supplementation effect on performance and intestinal epithelium \\ morphology in pigs reared in hot or cool environments
}

\section{Romo-Valdez Juan ${ }^{1 \mathrm{ID}}$, Barajas-Cruz Rubén ${ }^{1 \text { ID }}$, Enríquez-Verdugo Idalia ${ }^{1 \mathrm{ID}}$, Silva- Hidalgo Gabriela1 ${ }^{1 \mathrm{ID}}$, Güémez-Gaxiola Héctor ${ }^{1 \mathrm{ID}}$, Romo-Rubio Javier¹ ID}

${ }^{1}$ Facultad de Medicina Veterinaria y Zootecnia de la Universidad Autónoma de Sinaloa; Culiacán, Sinaloa, México; *Autor responsable y de correspondencia: Romo-Rubio Javier (romo60@uas.edu.mx) Facultad de Medicina Veterinaria y Zootecnia de la Universidad Autónoma de Sinaloa; Boulevard San Ángel s/n, Colonia San Benito, Culiacán de Rosales, Sinaloa, México; CP 80246. romo_14@hotmail.com, rubar@uas.edu.mx, enver@uas.edu.mx,gabsilhid@uas.edu.mx, hectorguem@gmail.com,romo60@uas.edu.mx

\section{RESUMEN}

Con el objetivo de determinar el efecto de la suplementación con metionina de zinc durante el periodo de gestación-lactación (GL) y desarrollo-finalización (DF) en el desempeño productivo y durante DF en la morfología del epitelio intestinal en cerdos criados en ambiente caluroso o fresco, se realizaron dos experimentos (Exp. 1, en la época calurosa; Exp. 2, en la época fresca del año). Se utilizaron 192 cerdos (96 por Exp.) con una edad promedio de $79 \mathrm{~d}$ y $26.39 \pm D E 4.97 \mathrm{~kg}$ de peso, hijos de cerdas que recibieron o no, alimento adicionado con $100 \mathrm{mg}$ de $\mathrm{Zn} / \mathrm{kg}$, a partir de los 80 a 114 días de gestación y durante $21 \mathrm{~d}$ de lactación (GL). En cada experimento, los cerdos fueron asignados a uno de cuatro tratamientos en un diseño completamente al azar con arreglo factorial $2 \times 2$. Los tratamientos fueron: T1 (Testigo; $n=24$ ), madres no suplementadas-cerdos no suplementados; T2 $(Z n D F ; n=24)$, madres no suplementadascerdos suplementados con $100 \mathrm{mg}$ de $\mathrm{Zn} / \mathrm{kg}$ alimento; T3 ( $\mathrm{Zn} \mathrm{GL;} \mathrm{n}=24)$, madres suplementadas-cerdos no suplementados y, T4 (Zn GL + Zn DF; n = 48), madres suplementadas + cerdos suplementados. La suplementación con $100 \mathrm{mg}$ de $\mathrm{Zn} / \mathrm{kg}$ de alimento durante GL y DF no modificó el desempeño productivo de los cerdos durante el periodo completo de estudio. La relación altura de la vellosidad: profundidad de la cripta fue mayor ( $p<0.01$ ) en los cerdos suplementados con Zn (3.36 vs. 2.77) durante la época de calor. Se observó una interacción $(\mathrm{p}<0.02)$ entre el clima y la suplementación con metionina de zinc en la profundidad de la cripta y en la relación V: C. La suplementación durante $G L$ tendió $(P=0.06)$ a bajar la mortalidad de los cerdos en desarrollo-finalización en la época fresca. Los resultados permiten concluir que la adición de metionina de zinc a la dieta mejora la integridad del epitelio intestinal en los cerdos en desarrollo-finalización criados bajo ambiente caluroso, y la suplementación durante el periodo de gestación y lactación reduce la mortalidad durante la engorda.

Palabras clave: Cerdo, Metionina de Zinc, Epitelio intestinal, Respuesta productiva.

\begin{abstract}
To determine the effect of zinc-methionine supplementation during the gestation-lactation (GL) and growingfinishing (DF) period in the performance and during DF on the epithelium intestinal morphology of fattening pigs under hot and cool condition, two experiments were carried out. The experiment (Exp.) 1 was carried out during the hot season and Exp. 2 during the cool season of the year. 192 pigs were used (96 per Exp.) with an average age of 79 days and $26.39 \pm S D 4.97 \mathrm{~kg}$ of body weight, piglets of sows that received or not feed added with $100 \mathrm{mg}$ of $\mathrm{Zn} / \mathrm{kg}$, from 80 to 114 days of gestation and during $21 \mathrm{~d}$ of lactation (LG). In each experiment, the pigs were assigned to one of four treatments in a completely random design with a 2
\end{abstract}


$x 2$ factorial arrangement. The treatments were: T1 (control, $n=24$ ), non-supplemented mothers-nonsupplemented pigs; T2 ( $Z n$ FD; $n=24)$, non-supplemented mothers, pigs supplemented with $100 \mathrm{mg}$ of Zn/kg feed; T3 (Zn LG; $n=24)$, supplemented mothers - non-supplemented pigs and, T4 (Zn LG + Zn DF; $\mathrm{n}=24$ ), supplemented mothers + supplemented pigs. Supplementation with $100 \mathrm{mg}$ of $\mathrm{Zn} / \mathrm{kg}$ of feed during GL and DF did not modify the productive performance of the pigs during the study period. However, the villus height: crypt depth ratio was higher $(p<0.01)$ in pigs supplemented with $\mathrm{Zn}(3.36$ vs. 2.77$)$ during the hot season. An interaction $(\mathrm{p}<0.02)$ between climate and zinc methionine supplementation was observed in the depth of the crypt and the V: $C$ ratio. Supplementation during $G L$ tended $(P=0.06)$ to lower the mortality of developing-finishing pigs in the cool season. According to results, it is concluded that the addition of zinc methionine to the diet improves the integrity of the intestinal epithelium in developing-finishing pigs reared under a hot environment, and supplementation during the gestation and lactation period reduces mortality during fattening.

Keywords: Pig, Zinc methionine, Intestinal epithelium, Productive response.

\section{INTRODUCCIÓN}

La producción animal se ve gravemente afectada por el estrés calórico (EC); se estima que la industria porcina de los Estados Unidos pierde más de $\$ 300$ millones de dólares al año; mientras que las pérdidas mundiales ascienden a miles de millones de dólares (St-Pierre et al., 2003). Las pérdidas económicas inducidas por el EC son el resultado de un rendimiento deficiente de las cerdas, un crecimiento reducido e inconsistente de los cerdos en engorda, una menor calidad de la canal y un aumento de los costos veterinarios (St-Pierre et al., 2003; Renaudeau et al., 2011). Las consecuencias del EC en el bajo desempeño productivo del cerdo pueden estar relacionadas con los efectos negativos sobre la integridad intestinal (Pearce et al., 2013a; Sanz-Fernandez et al., 2014).

Los mamíferos en EC redistribuyen la sangre a la periferia para maximizar la disipación de calor radiante, ocasionando un menor flujo sanguíneo y de nutrientes en el intestino; comprometiendo la barrera intestinal y aumentando su permeabilidad (Pearce et al., 2013b); además, el EC puede antagonizar con la digestibilidad y las vías anabólicas en los cerdos (Mani et al., 2012; Rakhshandeh et al., 2012). Debido a que los requerimientos de Zn se incrementan durante el estrés calórico (Lagana et al., 2007), se ha sugerido que la suplementación con Zn podría utilizarse para atenuar la disminución sérica del Zn durante periodos de altas temperaturas ambientales (Chand et al., 2014; Li et al., 2015). El Zinc en la forma de ion metálico divalente, $\mathrm{Zn}^{2+}$, es nutricionalmente esencial para todos los organismos vivos (Maret, 2013); es un mineral traza con probada importancia para la función de más de 300 enzimas (Chasapis et al., 2012). Las dietas para cerdos son generalmente complementadas con $\mathrm{Zn}$ inorgánico ( $\mathrm{ZnSO}^{4} \mathrm{u} \mathrm{ZnO}$ ), para asegurar el aporte requerido, siendo la fuente inorgánica de $\mathrm{ZnSO}_{4}$ la de mayor biodisponibilidad (NRC, 2012); sin embargo, en condiciones fisiológicas normales y con la ingesta adecuada, sólo del 5 al 15\% del Zn de la dieta es aparentemente absorbido (McDowell, 2003). Se ha sugerido que las fuentes orgánicas de $Z n$ son más biodisponibles que las formas inorgánicas, y la biodisponibilidad de las formas orgánicas respecto de las inorgánicas aumenta en presencia de antagonistas, como el $\mathrm{Ca}, \mathrm{P}$, ácido fítico y fibra cruda (Schlegel et al., 2013; Richards et al., 2015). Ming-Zhe et al. (2016), observaron 
que el valor biológico del zinc orgánico a partir de metionina de zinc (ZnMet), fue 64\% mayor que el del $\mathrm{ZnSO}_{4}$.

El objetivo del presente estudio fue determinar el efecto de la suplementación con metionina de zinc durante el periodo de gestación-lactación y el periodo de desarrollofinalización, en el desempeño productivo y durante el periodo de desarrollo-finalización en la morfología del epitelio intestinal de cerdos en engorda, criados bajo ambiente cálido o fresco.

\section{MATERIAL Y MÉTODOS}

Ubicación del área de estudio. El estudio se realizó en el área experimental para cerdos de engorda de la Facultad de Medicina Veterinaria y Zootecnia-UAS, ubicada en la granja porcina "La Huerta", localizada en el municipio de Culiacán, Sinaloa, México. El estudio consistió en dos experimentos; el experimento 1 se realizó durante los meses de julio a octubre (periodo cálido), y el experimento 2 en los meses de diciembre a marzo (periodo fresco). El análisis histológico de las muestras de intestino delgado (yeyuno, duodeno e íleon), tomadas en el rastro, fueron procesadas en el Laboratorio de Patología de la Facultad de Medicina Veterinaria y Zootecnia de la Universidad Autónoma de Sinaloa, de acuerdo con el procedimiento propuesto por Prophet et al. (1995).

Diseño experimental. Se utilizaron 192 cerdos (96 en cada experimento), con una edad

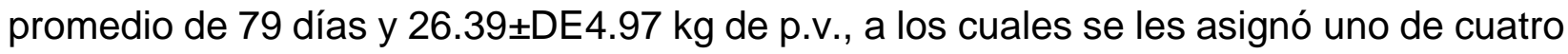
tratamientos en un diseño experimental completamente al azar, con arreglo factorial $2 \mathrm{x}$ 2. En cada experimento, los tratamientos consistieron en: T1 (Testigo; $n=24$ ), madres no suplementadas-cerdos no suplementados; T2 ( $\mathrm{Zn}$ DF; $\mathrm{n}=24)$, madres no suplementadas-cerdos suplementados con $100 \mathrm{mg}$ de Zn/kg MS; T3 (Zn GL; n = 24), madres suplementadas con $100 \mathrm{mg}$ de $\mathrm{Zn} / \mathrm{kg}$ MS-cerdos no suplementados y, T4 (Zn GL + Zn DF; $n=24$ ), madres suplementadas + cerdos suplementados.

Las dietas utilizadas se muestran en el cuadro 1. Los cerdos utilizados en el experimento fueron animales provenientes de un trabajo previo, hijos de cerdas (Líneas PIC ${ }^{\circledR}$ ) que recibieron o no alimento adicionado con metionina de zinc (100 mg/kg de MS) durante gestación (80-114 d) y lactancia (1-21 d); las dietas proporcionadas fueron con base en maíz-pasta de soya (cuadro 2). El zinc orgánico se proporcionó como metionina de zinc (ZnMet) de la premezcla Zinpro 120 (Zinproß 120; contiene 12\% de Zn y 27.3\% de metionina; Metionina de zinc Patente en EU No. 4,764,633 y 5,430,164; oficio de liberación México: B00.02.08.02.02.0398/11)

Manejo de los animales. Los cerdos de cada experimento, previamente pesados e identificados, fueron alojados en 12 corraletas, cada una con un espacio de $9 \mathrm{~m}^{2}(6 \times 1.5$ $\mathrm{m})$, con piso de concreto, equipados con comedero tipo tolva y bebedero de chupón integrado; en cada corraleta se alojaron 8 cerdos (4 machos y 4 hembras). Durante el periodo de prueba, que duró 90 días en cada experimento, los cerdos tuvieron acceso permanente a agua de bebida y alimentación a libre acceso. Se registró el alimento servido en cada corraleta y al final de cada experimento los cerdos fueron pesados y 
enviados al rastro, donde fueron sacrificados en rastro TIF de acuerdo con la NOM-033SAG/ZOO-2014. Después del sacrificio, fueron tomadas muestras de tejido del intestino delgado, de las porciones de duodeno, yeyuno e íleon, 15 cerdos de cada experimento; que recibieron o no alimento suplementado con $100 \mathrm{mg}$ de metionina de $\mathrm{zinc} / \mathrm{kg}$ de alimento durante el periodo de prueba.

Cuadro 1. Composición e información nutricional de las dietas utilizadas en la etapa de desarrollo y finalización

\begin{tabular}{|c|c|c|}
\hline Ingredientes & $\begin{array}{c}\text { Desarrollo } \\
\text { (30-60 kg de peso corporal) }\end{array}$ & $\begin{array}{r}\text { Finalizacić } \\
(60-100 \mathrm{~kg} \text { de pes } \\
\end{array}$ \\
\hline Maíz & 749 & 810 \\
\hline Pasta de soya & 217 & 165 \\
\hline Aceite & 9 & 5 \\
\hline \multirow[t]{2}{*}{ Premezcla mineral } & 25 & 20 \\
\hline & Aporte nutrimental & \\
\hline E.M.(Mcal Kg-1) & 3.351 & 3.353 \\
\hline Proteína (\%) & 16.702 & 14.688 \\
\hline Lisina (\%) & 1.052 & 0.875 \\
\hline Fibra (\%) & 2.524 & 2.520 \\
\hline Fósforo (\%) & 0.520 & 0.439 \\
\hline Calcio (\%) & 0.570 & 0.457 \\
\hline${ }^{\star} \operatorname{Zinc}\left(\mathrm{mg} \mathrm{kg}^{-1}\right)$ & 120.28 & 105.31 \\
\hline
\end{tabular}

${ }^{*}$ Contenido de Zn de la dieta testigo, aportado por la premezcla mineral como ZnO. La suplementación con $100 \mathrm{mg}$ de zinc se proporcionó como metionina de zinc (ZnMet) de la premezcla Zinpro120 (833.33 gr/ton de alimento en base seca). La dieta se elaboró con base en las tablas de requerimientos nutricionales para cerdos (NRC, 2012)

Cuadro 2. Composición e información nutricional de las dietas utilizadas en gestación y lactancia

\begin{tabular}{lll}
\hline Ingredientes & Gestación & Lactancia \\
\hline Maíz & 793 & 692 \\
Pasta de soya & 160 & 254 \\
Aceite & 5 & 18 \\
Premezcla mineral & 42 & 36 \\
& Aporte nutrimental & \\
E.M.(Mcal Kg-1) & 3.272 & 3.351 \\
Proteína (\%) & 14.165 & 17.953 \\
Lisina (\%) & 0.866 & 1.081 \\
Fibra (\%) & 2.463 & 2.492 \\
Fósforo (\%) & 0.596 & 0.699 \\
Calcio (\%) & 0.980 & 0.915
\end{tabular}

La suplementación con $100 \mathrm{mg}$ de zinc se proporcionó como metionina de zinc (ZnMet) de la premezcla Zinpro120 (833.33 gr/ton de alimento en base seca). La dieta se elaboró con base en las tablas de requerimientos nutricionales para cerdos (NRC, 2012)

Mediciones: Se registró semanalmente el alimento servido en cada corraleta; al final de cada experimento se pesaron los cerdos de cada corral y con la información de consumo de alimento y ganancia de peso se obtuvo la conversión alimenticia; se registró la mortalidad de cerdos en cada corral y con base en ello se determinó la tasa de mortalidad. 
Para el caso de la prueba de comportamiento productivo, la unidad experimental fue la corraleta.

Al finalizar cada experimento los cerdos fueron enviados al rastro, donde se tomaron muestras de cada segmento de intestino delgado (duodeno, yeyuno e íleon), 15 cerdos representativos de cada experimento (época calurosa y época fresca), que recibieron o no alimento suplementado con metionina de zinc; sin considerar si eran hijos de cerdas que recibieron o no alimento adicionado con metionina de zinc durante el periodo de gestación y lactancia. Las muestras fueron conservadas en frascos con formol amortiguado al $10 \%$ hasta su análisis histológico.

Determinación del THI. Los datos de temperatura $\left(\mathrm{t}^{\circ} \mathrm{C}\right)$ y humedad relativa $(\mathrm{HR}, \%)$ se tomaron con un termo higrómetro, ubicado dentro de la unidad experimental, y se registraron diariamente durante el periodo experimental (cuadros 3 y 4). El índice de temperatura y humedad $(\mathrm{THI})$, se calculó utilizando la fórmula $\mathrm{THI}=[0.8 \times$ temperatura ambiente $]+[(\% \mathrm{HR} / 100) \times$ (temperatura ambiente - 14.4) $]+46.4$ (Mader et al., 2006).

Cuadro 3. Índice de temperatura y humedad (THI), al que estuvieron expuestos los cerdos durante el periodo cálido (11 de julio al 08 de octubre de 2015)

\begin{tabular}{|c|c|c|c|c|c|c|c|}
\hline Semana & Prom. HR & $\begin{array}{c}\text { Temp. } \\
\text { Prom. }\left({ }^{\circ} \mathrm{C}\right)\end{array}$ & $\begin{array}{c}\text { Temp. } \\
\text { Max. }\left({ }^{\circ} \mathrm{C}\right)\end{array}$ & $\begin{array}{c}\text { Temp. } \\
\text { Min. }\left({ }^{\circ} \mathrm{C}\right)\end{array}$ & $\begin{array}{l}\mathrm{THI}^{1} \\
\text { Prom. }\end{array}$ & $\begin{array}{l}\text { THI } \\
\text { Max. }\end{array}$ & THI Min. \\
\hline 1 & 64.6 & 30.6 & 38.6 & 25.8 & 81.34 & 92.91 & 74.40 \\
\hline 2 & 77.3 & 28.0 & 31.9 & 25.1 & 79.31 & 85.44 & 74.75 \\
\hline 3 & 59.8 & 32.6 & 38.1 & 28.3 & 83.36 & 91.05 & 77.35 \\
\hline 4 & 64.1 & 31.7 & 36.5 & 27.5 & 82.84 & 89.76 & 76.79 \\
\hline 5 & 72.9 & 28.8 & 34.3 & 25.0 & 79.93 & 88.34 & 74.12 \\
\hline 6 & 76.4 & 28.7 & 34.1 & 25.6 & 80.28 & 88.73 & 75.43 \\
\hline 7 & 73.2 & 29.8 & 36.9 & 24.9 & 81.51 & 92.39 & 73.76 \\
\hline 8 & 75.2 & 29.5 & 36.2 & 25.1 & 81.35 & 91.75 & 77.18 \\
\hline 9 & 78.1 & 29.0 & 35.3 & 24.8 & 81.39 & 95.54 & 74.36 \\
\hline 10 & 77.9 & 28.9 & 35.0 & 24.8 & 80.81 & 90.44 & 74.34 \\
\hline 11 & 73.4 & 30.3 & 37.4 & 25.8 & 82.31 & 93.20 & 75.40 \\
\hline 12 & 72.0 & 30.6 & 39.0 & 25.5 & 82.54 & 84.94 & 74.79 \\
\hline 13 & 79.1 & 26.1 & 32.3 & 22.3 & 76.53 & 86.40 & 71.28 \\
\hline Promedio & 72.61 & 29.58 & 35.81 & 25.42 & 81.03 & 90.06 & 74.92 \\
\hline
\end{tabular}

Histología intestinal. Las muestras fueron remitidas al Laboratorio de Patología de la Facultad de Medicina Veterinaria y Zootecnia de la Universidad Autónoma de Sinaloa, para su procesamiento mediante la técnica de rutina de inclusión en parafina y posterior tinción con la técnica de hematoxilina y eosina. Para la evaluación histológica se utilizó un microscopio binocular digital marca Motic ${ }^{\circledR}$ modelo BA 201, equipado con cámara y software para foto-documentar imágenes; se tomaron imágenes de 30 campos de cada 
sección intestinal, se midió la longitud (en micras) de 10 vellosidades; así como la profundidad (en micras) de las criptas, y con base en ello se calculó la altura promedio de las vellosidades de cada sección intestinal; así como de la profundidad de la cripta. Con base en esta información se determinó la relación altura de vellosidad, profundidad de la cripta ( $\mathrm{V}: \mathrm{C})$; lo que se utilizó para el análisis estadístico correspondiente. Cabe mencionar que las muestras de cada sección del intestino delgado que no cumplieron con los requisitos para su análisis histológico, se desecharon y no fueron consideradas en el análisis estadístico.

Cuadro 4. Índice de temperatura y humedad (THI), al que estuvieron expuestos los cerdos durante el periodo fresco (02 de diciembre de 2015 al 01 de marzo de 2016)

\begin{tabular}{|c|c|c|c|c|c|c|c|}
\hline Semana & Prom. HR & $\begin{array}{c}\text { Temp. } \\
\text { Prom. }\left({ }^{\circ} \mathrm{C}\right)\end{array}$ & $\begin{array}{c}\text { Temp. } \\
\text { Max. }\left({ }^{\circ} \mathrm{C}\right)\end{array}$ & $\begin{array}{c}\text { Temp. } \\
\text { Min. }\left({ }^{\circ} \mathrm{C}\right)\end{array}$ & $\begin{array}{l}\text { Prom. } \\
\text { THI }^{1}\end{array}$ & $\begin{array}{c}\text { THI } \\
\text { Max. }\end{array}$ & THI Min. \\
\hline 1 & 64.6 & 22.7 & 33.3 & 15.3 & 70.36 & 85.24 & 59.22 \\
\hline 2 & 74.8 & 19.3 & 27.9 & 12.5 & 65.50 & 78.81 & 54.90 \\
\hline 3 & 72.7 & 19.0 & 26.8 & 13.1 & 64.94 & 76.85 & 55.93 \\
\hline 4 & 64.9 & 17.2 & 26.6 & 9.8 & 61.97 & 75.59 & 51.25 \\
\hline 5 & 66.8 & 17.3 & 26.1 & 10.0 & 62.17 & 75.09 & 48.00 \\
\hline 6 & 64.4 & 20.0 & 29.7 & 13.6 & 66.00 & 80.01 & 56.76 \\
\hline 7 & 72.2 & 20.1 & 27.5 & 14.6 & 66.59 & 77.85 & 58.22 \\
\hline 8 & 72.7 & 19.6 & 27.5 & 13.8 & 65.86 & 77.92 & 57.00 \\
\hline 9 & 76.2 & 28.8 & 28.9 & 14.9 & 80.41 & 80.57 & 58.70 \\
\hline 10 & 74.4 & 20.0 & 30.2 & 12.7 & 66.56 & 82.31 & 55.29 \\
\hline 11 & 80.6 & 21.4 & 28.5 & 16.8 & 69.16 & 80.56 & 62.24 \\
\hline 12 & 77.3 & 22.0 & 31.0 & 15.5 & 69.87 & 84.03 & 59.65 \\
\hline 13 & 74.3 & 20.1 & 29.2 & 13.1 & 66.71 & 80.75 & 55.58 \\
\hline Promedio & 72.0 & 20.57 & 28.70 & 13.5 & 67.39 & 79.66 & 56.36 \\
\hline
\end{tabular}

Análisis estadístico. A los datos obtenidos de consumo diario de alimento (CDA), ganancia diaria de peso (GDP), conversión alimenticia (CA), ganancia de peso total por etapa (GPT) y mortalidad durante el experimento; así como, a la relación vellosidad, cripta; se les aplicó un análisis de varianza para un diseño completamente al azar, con arreglo factorial (Steel y Torrie, 1985); fijándose un alfa máximo de 0.05 para aceptar diferencia estadística.

Para el análisis del desempeño productivo el modelo matemático, fue el siguiente: $Y_{i j k}=$ $\mu+Z n_{i}+A_{j}+Z n A_{i j}+E_{i j k ;}$ donde: $Y_{i j k}=$ es la variable de respuesta, $\mu=$ es la media general del experimento, $Z n_{i}=$ es el efecto del $i$-ésimo nivel de Zinc, $A_{j}=$ el efecto del $j$ ésimo factor de alimentación, $Z n^{*} A_{i j}=$ efecto de la interacción y $E_{i j k}=$ es el error aleatorio. 
Para el análisis de la morfología intestinal, el modelo matemático fue el siguiente: $Y_{i j k}=$ $\mu+Z n_{i}+C_{j}+Z n C_{i j}+E_{i j k}$; donde: $Y_{i j k}=$ es la variable de respuesta, $\mu=$ es la media general del experimento, $Z n_{i}=$ es el efecto del $i$-ésimo nivel de Zinc, $C_{j}=$ el efecto del $j$ ésimo factor de clima, $Z n^{*} C_{i j}=$ efecto de la interacción y $E_{i j k}=$ es el error aleatorio.

\section{RESULTADOS Y DISCUSIÓN}

Experimento 1. Los resultados del efecto del consumo de alimento adicionado con metionina de Zn (ZnMet) sobre el desempeño productivo de los cerdos durante la etapa de desarrollo-finalización, en condiciones de alta carga calórica, se muestran en el cuadro 5. En la etapa de finalización (día 50 al 90, del periodo experimental), la GDP tendió ( $p=$ $0.07)$ a mejorar y el CDA fue mayor $(p=0.03)$ en los cerdos hijos de cerdas suplementadas con Zn durante la $G L$; observándose una tendencia $(P=0.10)$ de mejora en la ganancia de peso total durante esta etapa; sin embargo, al analizar el periodo completo de estudio (90 d), la suplementación con metionina de zinc no mejoró el desempeño productivo de los cerdos.

Los resultados del efecto del consumo de alimento adicionado con ZnMet en la morfología epitelial del intestino delgado durante la época de calor y época fresca, se muestran en los Cuadros 7 y 8 . Los resultados indican que los cerdos que consumieron dietas suplementadas con $100 \mathrm{mg}$ de $\mathrm{Zn} / \mathrm{kg}$ de alimento, a partir de metionina de zinc, durante la época calurosa, tuvieron una mayor $(p<0.01)$ relación $\mathrm{V}$ : C (3.36 vs 2.77$)$, respecto de los que no consumieron zinc adicional durante dicho periodo. El consumo de zinc adicional disminuyó $(p<0.05)$ la profundidad de las criptas en todos los segmentos (duodeno, yeyuno e íleon) del intestino delgado.

Experimento 2. Durante la época fresca del año los tratamientos no modificaron la respuesta productiva del cerdo (cuadro 6), ni la morfología del epitelio intestinal (cuadros 7 y 8); sin embargo, el consumo adicional de metionina de zinc durante el periodo de gestación-lactación, tendió $(p=0.06)$ a disminuir la mortalidad durante el periodo de engorda. Al respecto, se ha informado que la suplementación con $\mathrm{Zn}$ en la dieta de la cerda durante la gestación y lactancia, reduce la mortalidad predestete (Payne et al., 2006; Romo et al., 2017) y mejora la función inmune de los lechones destetados (Romo et al., 2017); lo que concuerda con los resultados obtenidos en el presente estudio.

La cantidad de Zn orgánico (ZnMet) suplementado a los cerdos en el presente estudio fue de $100 \mathrm{mg} / \mathrm{kg}$ de alimento, adicionales al contenido de Zn inorgánico aportado por las dietas (120.20 mg/kg en la dieta de desarrollo y $105.31 \mathrm{mg} / \mathrm{kg}$ en la de finalización), cantidad más alta que la recomendada por el NRC (2012); sin embargo, estos niveles no son tóxicos y son de uso rutinario en dietas porcinas comerciales.

Los cerdos del exp. 2 estuvieron expuestos a una temperatura promedio de $20.57^{\circ} \mathrm{C}$ y humedad relativa de 72.0\% (cuadro 4; CIAD, 2015, 2016); permaneciendo en un THI de 
67.39, condiciones ambientales bajo las cuales el cerdo no sufre tensión fisiológica derivada del ambiente físico (Mader et al., 2006). En tanto que los cerdos del experimento 1, permanecieron en un THI de 81 puntos (en peligro fisiológico, ver cuadro 3); con una temperatura ambiental promedio de $29.58^{\circ} \mathrm{C}$ y $72.61 \%$ de humedad relativa. La diferencia en la temperatura ambiental y humedad relativa entre los cerdos del experimento 1 , respecto de los del experimento 2 , fue de $9.01^{\circ} \mathrm{C}$ y $0.61 \%$.

Cuadro 5. Efecto del consumo de alimento adicionado con $100 \mathrm{ppm}$ de Zinc orgánico en el desempeño productivo de cerdos en desarrollo-finalización, durante época calurosa (julio-octubre)

\begin{tabular}{|c|c|c|c|c|c|c|c|c|}
\hline \multirow[t]{3}{*}{ Variable } & \multirow{2}{*}{\multicolumn{4}{|c|}{ Tratamientos }} & \multirow[t]{3}{*}{ EEM $^{1}$} & \multicolumn{2}{|c|}{$\begin{array}{l}\text { Efectos } \\
\text { principales }\end{array}$} & Interacción \\
\hline & & & & & & \multicolumn{3}{|c|}{ Valores de $\mathrm{P}$} \\
\hline & Testigo & Zn DF & Zn GL & $\begin{array}{l}\text { Zn GL + } \\
\text { Zn DF }\end{array}$ & & $\begin{array}{l}\mathrm{Zn} \\
\mathrm{GL}\end{array}$ & $\begin{array}{l}\mathrm{Zn} \\
\mathrm{DF}\end{array}$ & $\begin{array}{l}\mathrm{Zn} \mathrm{GL}{ }^{*} \\
\mathrm{Zn} \mathrm{DF}\end{array}$ \\
\hline Observaciones (n) & 3 & 3 & 3 & 3 & & & & \\
\hline Cerdos (n) & 24 & 24 & 24 & 24 & & & & \\
\hline Periodo (días) & 90 & 90 & 90 & 90 & & & & \\
\hline Peso inicial (kg) & 21.63 & 22.33 & 21.73 & 21.50 & 0.7445 & & & \\
\hline Peso a $49 \mathrm{~d}(\mathrm{~kg})$ & 54.16 & 55.70 & 56.67 & 52.13 & 1.9154 & 0.90 & 0.73 & 0.50 \\
\hline Ganancia de peso a $49 \mathrm{~d}(\mathrm{Kg})$ & 32.533 & 33.333 & 34.933 & 30.633 & 1.3550 & 0.96 & 0.57 & 0.41 \\
\hline $\mathrm{GDP}^{2}(\mathrm{~kg})$ & 0.663 & 0.681 & 0.713 & 0.626 & 0.0277 & 0.96 & 0.58 & 0.41 \\
\hline $\mathrm{CDA}^{3}(\mathrm{~kg})$ & 1.603 & 1.596 & 1.650 & 1.570 & 0.0539 & 0.93 & 0.73 & 0.77 \\
\hline$C A^{4}$ & 2.426 & 2.353 & 2.310 & 2.533 & 0.0395 & 0.66 & 0.32 & 0.07 \\
\hline Peso a $90 \mathrm{~d}(\mathrm{~kg})$ & 79.50 & 79.17 & 83.07 & 79.67 & 1.9142 & 0.65 & 0.68 & 0.73 \\
\hline $\begin{array}{l}\text { Ganancia de peso de } 50-90 \mathrm{~d} \\
(\mathrm{~kg})\end{array}$ & 25.667 & 23.467 & 26.400 & 27.533 & 0.7091 & 0.10 & 0.69 & 0.23 \\
\hline $\mathrm{GDP}^{2}(\mathrm{~kg})$ & 0.591 & 0.568 & 0.628 & 0.696 & 0.0222 & 0.07 & 0.57 & 0.27 \\
\hline $\mathrm{CDA}^{3}(\mathrm{~kg})$ & 1.907 & 1.773 & 2.033 & 1.990 & 0.0416 & 0.03 & 0.23 & 0.52 \\
\hline $\mathrm{CA}^{4}$ & 3.230 & 3.133 & 3.240 & 2.923 & 0.0910 & 0.62 & 0.31 & 0.58 \\
\hline $\begin{array}{l}\text { Ganancia de peso en el } \\
\text { periodo completo }(\mathrm{kg})\end{array}$ & 57.700 & 56.800 & 61.333 & 58.167 & 1.4036 & 0.44 & 0.52 & 0.72 \\
\hline $\mathrm{GDP}^{2}(\mathrm{~kg})$ & 0.641 & 0.631 & 0.681 & 0.646 & 0.0156 & 0.44 & 0.52 & 0.72 \\
\hline $\mathrm{CDA}^{3}(\mathrm{~kg})$ & 1.763 & 1.706 & 1.850 & 1.783 & 0.0402 & 0.37 & 0.50 & 0.95 \\
\hline $\mathrm{CA}^{4}$ & 2.760 & 2.706 & 2.713 & 2.756 & 0.227 & 0.97 & 0.92 & 0.36 \\
\hline Muertos & 0.33 & 0.667 & 0 & 0.667 & 0.1930 & 0.69 & 0.25 & 0.69 \\
\hline
\end{tabular}

Tratamientos: Testigo $=$ madres no suplementadas-cerdos no suplementados, ZnDF = Madres no suplementadas-cerdos suplementados; ZnGL = madres suplementadas-cerdos no suplementados; ZnGL + ZnDF = madres suplementadas-cerdos suplementados. Suplemento de100 mg de Zn/kg de alimento, proporcionados a partir de metionina de zinc (Zinpro 100; Zinpro, Eden Praire, MN). Dos Factores: método de suplementación = ZnGL y ZnCF, nivel de adición de Zn (0 y $100 \mathrm{mg} / \mathrm{kg}$ alimento); ${ }^{1} \mathrm{Error}$ estándar de la media; ${ }^{2}$ Ganancia diaria de peso corporal; ${ }^{3}$ Consumo diario de alimento y ${ }^{4}$ Conversión alimenticia

Los resultados del análisis morfológico de las muestras de duodeno, yeyuno e íleon, muestran que los cerdos que consumieron dietas suplementadas con $100 \mathrm{mg}$ de $\mathrm{Zn} / \mathrm{kg}$ de alimento, a partir de metionina de zinc, durante la época calurosa, tuvieron una mayor $(p<0.01)$ relación $\mathrm{V}: \mathrm{C}(3.36$ vs 2.77$)$, respecto de los que no consumieron zinc adicional durante dicho periodo. El consumo de zinc adicional disminuyó $(p<0.05)$ la profundidad de las criptas en todos los segmentos (duodeno, yeyuno e íleon) del intestino delgado. Se observó una interacción $(p<0.02)$ entre el clima y la suplementación con metionina de zinc en la profundidad de la cripta y en la relación V: C. El efecto del clima y la 
suplementación con metionina de zinc en la morfología epitelial de cada segmento del intestino delgado analizado, se describe a continuación:

Cuadro 6. Efecto del consumo de alimento adicionado con 100 ppm de Zinc orgánico en el desempeño productivo de cerdos en desarrollo-finalización, durante época fresca (diciembre a marzo)

\begin{tabular}{|c|c|c|c|c|c|c|c|c|}
\hline \multirow[t]{3}{*}{ Variable } & \multirow{2}{*}{\multicolumn{4}{|c|}{ Tratamientos }} & \multirow[t]{3}{*}{ EEM $^{1}$} & \multicolumn{2}{|c|}{$\begin{array}{c}\text { Efectos } \\
\text { principales }\end{array}$} & \multirow{2}{*}{$\begin{array}{l}\text { Interacci } \\
\text { ón }\end{array}$} \\
\hline & & & & & & \multicolumn{2}{|c|}{ Valores de $\mathrm{P}$} & \\
\hline & Testigo & Zn DF & Zn GL & $\begin{array}{l}\mathrm{Zn} \mathrm{GL} \mathrm{+} \\
\mathrm{Zn} \mathrm{DF}\end{array}$ & & $\begin{array}{l}\mathrm{Zn} \\
\mathrm{GL}\end{array}$ & $\begin{array}{l}\mathrm{Zn} \\
\mathrm{DF}\end{array}$ & $\begin{array}{c}\mathrm{Zn} \mathrm{GL}{ }^{*} \\
\mathrm{Zn} \mathrm{DF}\end{array}$ \\
\hline Observaciones (n) & 3 & 3 & 3 & 3 & & & & \\
\hline Cerdos (n) & 24 & 24 & 24 & 24 & & & & \\
\hline Periodo (días) & 90 & 90 & 90 & 90 & & & & \\
\hline Peso inicial (kg) & 32.13 & 30.00 & 29.93 & 31.86 & 0.8132 & & & \\
\hline Peso a 49 d (kg) & 69.60 & 71.40 & 67.36 & 72.03 & 1.4980 & 0.81 & 0.35 & 0.67 \\
\hline Ganancia de peso a $49 \mathrm{~d}(\mathrm{Kg})$ & 37.467 & 41.400 & $\begin{array}{c}37.43 \\
3\end{array}$ & 40.133 & 1.2656 & 0.81 & 0.25 & 0.82 \\
\hline $\mathrm{GDP}^{1}(\mathrm{~kg})$ & 0.764 & 0.844 & 0.764 & 0.818 & 0.0258 & 0.81 & 0.26 & 0.82 \\
\hline $\mathrm{CDA}^{2}(\mathrm{~kg})$ & 1.980 & 2.030 & 2.043 & 2.076 & 0.0326 & 0.47 & 0.58 & 0.91 \\
\hline $\mathrm{CA}^{3}$ & 2.603 & 2.413 & 2.686 & 2.580 & 0.0723 & 0.44 & 0.36 & 0.79 \\
\hline Peso a $90 \mathrm{~d}(\mathrm{~kg})$ & 107.83 & 112.23 & $\begin{array}{c}104.2 \\
9\end{array}$ & 109.29 & 1.9853 & 0.46 & 0.29 & 0.94 \\
\hline $\begin{array}{l}\text { Ganancia de peso de } 50-90 \mathrm{~d} \\
(\mathrm{~kg})\end{array}$ & 38.35 & 40.83 & 36.92 & 37.26 & 0.8972 & 0.20 & 0.46 & 0.57 \\
\hline $\mathrm{GDP}^{1}(\mathrm{~kg})$ & 0.910 & 0.972 & 0.879 & 0.888 & 0.0213 & 0.21 & 0.43 & 0.55 \\
\hline $\mathrm{CDA}^{2}(\mathrm{~kg})$ & 3.030 & 3.016 & 2.850 & 2.863 & 0.0568 & 0.20 & 1.00 & 0.91 \\
\hline $\mathrm{CA}^{3}$ & 3.326 & 3.106 & 3.270 & 3.226 & 0.0634 & 0.82 & 0.36 & 0.53 \\
\hline $\begin{array}{l}\text { Ganancia de peso en el } \\
\text { periodo completo }(\mathrm{kg})\end{array}$ & 75.700 & 82.267 & $\begin{array}{c}74.13 \\
3\end{array}$ & 77.400 & 1.9356 & 0.46 & 0.29 & 0.94 \\
\hline $\mathrm{GDP}^{1}(\mathrm{~kg})$ & 0.841 & 0.914 & 0.826 & 0.859 & 0.0211 & 0.45 & 0.26 & 0.66 \\
\hline $\mathrm{CDA}^{2}(\mathrm{~kg})$ & 2.493 & 2.513 & 2.443 & 2.466 & 0.0334 & 0.54 & 0.78 & 0.98 \\
\hline $\mathrm{CA}^{3}$ & 2.963 & 2.760 & 2.976 & 2.876 & 0.0497 & 0.53 & 0.16 & 0.61 \\
\hline Muertos & 0.67 & 0.33 & 0 & 0 & 0.1306 & 0.06 & 0.50 & 0.50 \\
\hline
\end{tabular}

Tratamientos: Testigo $=$ madres no suplementadas-cerdos no suplementados, ZnDF $=$ Madres no suplementadas-cerdos suplementados; ZnGL = madres suplementadas-cerdos no suplementados; ZnGL + ZnDF = madres suplementadas-cerdos suplementados. Suplemento de100 mg de Zn/kg de alimento, proporcionados a partir de metionina de zinc (Zinpro 100; Zinpro, Eden Praire, MN). Dos Factores: método de suplementación = ZnGL y ZnCF, nivel de adición de Zn (0 y $100 \mathrm{mg} / \mathrm{kg}$ alimento); ${ }^{1} \mathrm{Error}$ estándar de la media; ${ }^{2}$ Ganancia diaria de peso corporal; ${ }^{3}$ Consumo diario de alimento y ${ }^{4}$ Conversión alimenticia

Morfología epitelial del duodeno. El análisis indica una interacción $(p<0.01)$ del clima con el zinc, observándose que en clima fresco los cerdos tienen una mejor respuesta al consumo adicional de $100 \mathrm{mg}$ de $\mathrm{Zn} / \mathrm{kg}$ de alimento, con una mayor altura de las vellosidades respecto de los cerdos suplementados durante la época calurosa. En el caso de la profundidad de las criptas, los cerdos que consumieron alimento adicionado con metionina de zinc, tanto en época cálida como en época de fresca, tuvieron una menor $(p<0.02)$ profundidad; en tanto que la relación $\mathrm{V}: C$ fue influenciada $(p<0.01)$ por el clima; siendo mayor durante la época fresca. 
Morfología epitelial del yeyuno. El clima fue el factor que modificó $(p<0.01)$ la altura de las vellosidades, observándose una mayor altura de estas en los cerdos tratados durante la época fresca; sin embargo, la profundidad de las criptas fue menor $(p<0.05)$ en los cerdos que consumieron alimento adicionado con metionina de zinc, tanto en la época cálida como en la fresca, así como en los que no consumieron zinc adicional durante la época fresca $(p<0.05)$. De igual manera, la mayor $(p<0.03)$ relación $V: C$ se observó en los animales y épocas del año referidas previamente.

Morfología epitelial del íleon. En la región del íleon la altura de las vellosidades también fue mayor $(p<0.01)$ en los en los cerdos tratados durante la época fresca; sin embargo, la profundidad de la cripta fue menor $(p<0.03)$ en los cerdos que consumieron alimento adicionado con metionina de zinc, tanto en la época cálida y como en la época fresca, observándose una interacción $(p<0.03)$ entre los factores clima y zinc. Se encontró una interacción $(p<0.04)$ entre clima y zinc en la relación $\mathrm{V}$ : C.

Cuadro 7. Influencia del clima y la suplementación con zinc en la morfología de las diferentes regiones del intestino delgado, en cerdos en engorda

\begin{tabular}{|c|c|c|c|c|c|c|c|c|c|c|c|}
\hline \multirow[t]{3}{*}{ Variables } & \multicolumn{4}{|c|}{ Época de Calor } & \multicolumn{4}{|c|}{ Época Fresca } & \multirow{2}{*}{\multicolumn{2}{|c|}{$\begin{array}{c}\text { Factores } \\
\text { Principales }\end{array}$}} & \multirow{3}{*}{$\begin{array}{c}\text { Interacción } \\
\mathrm{C}^{\star} \mathrm{Z}\end{array}$} \\
\hline & \multicolumn{2}{|c|}{ Cerdos - Zn } & \multicolumn{2}{|c|}{ Cerdos $+\mathrm{Zn}$} & \multicolumn{2}{|c|}{ Cerdos - Zn } & \multicolumn{2}{|c|}{ Cerdos + Zn } & & & \\
\hline & Media & $\pm E E$ & Media & $\pm \mathrm{EE}$ & Media & $\pm \mathrm{EE}$ & Media & $\pm E E$ & Clima & Zinc & \\
\hline \multicolumn{12}{|l|}{ Duodeno } \\
\hline Cerdos, $\mathrm{n}$ & & 14 & & 13 & & 10 & & 15 & & & \\
\hline Alto $\mathrm{V}, \mu \mathrm{m}$ & 391 & $15.90 \mathrm{~b}$ & 327 & $15.97 \mathrm{c}$ & 423 & $17.86 \mathrm{ab}$ & 451 & $14.58 b$ & $<0.01$ & 0.26 & $<0.01$ \\
\hline Profundo $C, \mu \mathrm{m}$ & 153 & $7.88 \mathrm{a}$ & 115 & $8.18 b$ & 121 & $9.33 a b$ & 116 & $7.62 b$ & 0.07 & 0.02 & 0.06 \\
\hline Relación V:C & 2.70 & $0.190 c$ & 2.98 & $0.197 \mathrm{bc}$ & 3.69 & $0.215 a b$ & 3.90 & $0.190 \mathrm{a}$ & $<0.01$ & 0.24 & 0.86 \\
\hline \multicolumn{12}{|l|}{ Yeyuno } \\
\hline Cerdos, $\mathrm{n}$ & & 15 & & 13 & & 10 & & 15 & & & \\
\hline AltoV, $\mu \mathrm{m}$ & 383 & $12.60 b$ & 408 & $13.54 a b$ & 458 & $15.44 a$ & 452 & $12.60 \mathrm{a}$ & $<0.01$ & 0.51 & 0.25 \\
\hline Profundo $C, \mu \mathrm{m}$ & 136 & $6.24 a$ & 111 & $6.71 \mathrm{ab}$ & 111 & 7.64ab & 108 & $6.24 b$ & 0.05 & 0.05 & 0.13 \\
\hline $\begin{array}{l}\text { Relación V:C } \\
\text { Íleon }\end{array}$ & 2.97 & $0.190 \mathrm{~b}$ & 3.80 & $0.204 a$ & 4.18 & $0.222 \mathrm{a}$ & 4.25 & $0.197 \mathrm{a}$ & $<0.01$ & 0.03 & 0.07 \\
\hline Cerdos, $\mathrm{n}$ & & 13 & & 14 & & 9 & & 15 & & & \\
\hline Alto $\mathrm{V}, \mu \mathrm{m}$ & 363 & $14.03 b$ & 351 & $13.52 b$ & 423 & $16.86^{a}$ & 435 & $13.06 \mathrm{a}$ & $<0.01$ & 0.98 & 0.43 \\
\hline Profundo $C, \mu \mathrm{m}$ & 140 & $6.31 a$ & 110 & $6.38 b$ & 112 & $7.58 b$ & 113 & $5.87 \mathrm{~b}$ & 0.07 & 0.03 & 0.03 \\
\hline Relación V:C* & 2.66 & $0.16 b$ & 3.30 & $0.15 a$ & 3.95 & $0.187 a$ & 3.86 & $0.15 a$ & $<0.01$ & 0.11 & 0.04 \\
\hline
\end{tabular}

${ }^{*}$ Relación $\mathrm{V}: \mathrm{C}$ indica el producto de la división de la altura de vellosidad entre profundidad de cripta.

a b, c, indica diferencia estadística (Tuckey, $P<0.05$ )

Al hacer el análisis agrupado (ver cuadro 8) de la morfología epitelial de los tres segmentes del intestino delgado, se observó que el clima tiene un mayor efecto $(p<0.01)$ sobre la altura de las vellosidades, y que existe una interacción $(p<0.02)$ entre el clima y la suplementación con zinc en la reducción de la profundidad de las criptas y en la elevación de la relación $\mathrm{V}$ : C. 
Cuadro 8. Influencia del clima y la adición de metionina de zinc a la dieta en la morfología intestinal de cerdos en engorda (valores combinados de las tres regiones: Duodeno, yeyuno e íleon)

\begin{tabular}{|c|c|c|c|c|c|c|c|c|c|c|c|}
\hline \multirow{3}{*}{ Variables } & \multicolumn{4}{|c|}{ Época de Calor } & \multicolumn{4}{|c|}{ Época Fresca } & \multirow{2}{*}{\multicolumn{2}{|c|}{$\begin{array}{c}\text { Factores } \\
\text { Principales }\end{array}$}} & \multirow{3}{*}{$\begin{array}{c}\text { Interacción } \\
C^{\star} Z\end{array}$} \\
\hline & \multicolumn{2}{|c|}{ Cerdos - Zn } & \multicolumn{2}{|c|}{ Cerdos $+\mathrm{Zn}$} & \multicolumn{2}{|c|}{ Cerdos - Zn } & \multicolumn{2}{|c|}{ Cerdos $+\mathrm{Zn}$} & & & \\
\hline & Media & $\pm \mathrm{EE}$ & Media & $\pm \mathrm{EE}$ & Media & $\pm E E$ & Media & $\pm E E$ & Clima & Zinc & \\
\hline \multicolumn{12}{|l|}{ Intestino delgado } \\
\hline Cerdos, $\mathrm{n}$ & \multicolumn{2}{|c|}{15} & \multicolumn{2}{|c|}{14} & \multicolumn{2}{|c|}{10} & \multicolumn{2}{|c|}{15} & & & \\
\hline Muestras, n & \multicolumn{2}{|c|}{42} & \multicolumn{2}{|c|}{40} & \multicolumn{2}{|c|}{29} & \multicolumn{2}{|c|}{45} & & & \\
\hline Alto $\mathrm{V}, \mu \mathrm{m}$ & 378 & $8.213 b$ & 362 & $8.414 b$ & 436 & $9.467 a$ & 446 & $8.210 a$ & $<0.01$ & 0.68 & 0.13 \\
\hline Profundo $\mathrm{C}, \mu \mathrm{m}$ & 143 & $3.897 a$ & 112 & $3.992 b$ & 113 & $4.463 b$ & 113 & $3.895 b$ & $<0.01$ & $<0.01$ & $<0.01$ \\
\hline Relación V:C* & 2.77 & $0.105 \mathrm{c}$ & 3.36 & $0.107 b$ & 3.94 & $0.119 a$ & 4.00 & $0.105 a$ & $<0.01$ & $<0.01$ & 0.02 \\
\hline
\end{tabular}

${ }^{*}$ Relación V:C, indica el producto de la división de la altura de vellosidad entre profundidad de cripta.

a b, c, indica diferencia estadística (Tuckey, $P<0.01$ )

Los resultados del presente estudio indican que el zinc suplementario a nivel de 100 $\mathrm{mg} / \mathrm{kg}$ de alimento, a partir de metionina de zinc, mejora la morfología intestinal de cerdos en engorda criados bajo ambiente caluroso; pero cuando se suplementa a cerdos criados bajo ambientes frescos se mejora la respuesta.

Se ha sugerido que una baja relación V: C puede indicar una atrofia de las vellosidades, asociada con un aumento en la tasa de pérdida de células del vértice de las vellosidades, que concurre con una mayor producción de células en las criptas y, por lo tanto, una mayor profundidad de éstas. Una mayor proporción de V: C, sugiere un estado más sano del intestino (Tang et al., 1999). Al et al. (2015) indicaron que la altura de las vellosidades y la profundidad de la cripta muestran cambios de desarrollo notablemente interdependientes; por lo tanto, su medida morfométrica no puede considerarse individualmente; en su lugar debe evaluarse la proporción de V: C; lo que sugiere, que la mayor proporción V: C observada en el presente estudio (3.36 vs. 2.77; $p<0.01$ ) en los cerdos que recibieron suplementación con $100 \mathrm{mg}$ de $\mathrm{Zn}$ (ZnMet) kg-1 durante la época de calor, respecto de los que no recibieron zinc adicional, se debe a la acción benéfica del Zn orgánico sobre el mantenimiento de la integridad y la morfología intestinal. Estos resultados son congruentes con los obtenidos por Bouwhuis et al. (2016), quienes informaron que la inclusión de metionina de zinc aumentó la altura de la vellosidad en el yeyuno, pero no aumentó la ganancia de peso de los cerdos. Además, estudios más recientes demostraron que la suplementación con zinc (450 $\mathrm{mg} \mathrm{kg}^{-1}$ de nano-ZnO y 3,000 $\mathrm{mg} \mathrm{kg}^{-1}$ de $\mathrm{ZnO}$ ) protegen la morfología del intestino delgado, al aumentar la altura de las vellosidades (Long et al., 2017; Pei et al., 2018). Por su parte, Li et al. (2001), observaron que los cerdos que recibieron $\mathrm{ZnO}$ suplementario tuvieron mayor grosor de la mucosa y altura de la vellosidad, en los sitios proximal y medial del intestino delgado, con respecto a los cerdos que recibieron la dieta testigo. 
Se sabe que el estrés calórico afecta la salud y función gastrointestinal (Eshel et al., 2001), ya que durante el estrés calórico el flujo de sangre se desvía del sistema esplénico a la piel, en un intento de disipar el exceso de calor (Lambert, 2009); un flujo sanguíneo reducido e hipertermia conducen a hipoxia, estrés oxidativo y nitrosante en el enterocito (Pearce et al., 2013a, b, c), dañando las membranas celulares y mucosas; así como las uniones estrechas, aumentando la permeabilidad intestinal (Lambert et al., 2002). Al respecto, Pearce et al. (2015) observaron que el estrés calórico aumenta el paso de sustancias de alto peso molecular y endotoxinas circulantes; además de un aumento en la autolisis del epitelio intestinal.

Se ha demostrado que la suplementación con óxido de zinc reduce la permeabilidad intestinal en lechones al destete, al tiempo que aumenta la cantidad y expresión de proteínas de unión estrecha (Zhang y Guo, 2009). También niveles crecientes del complejo de zinc AA $(220$ y $320 \mathrm{mg} / \mathrm{kg}$ ) aumentaron la integridad del tracto gastrointestinal, en comparación con cerdos testigo que recibieron $120 \mathrm{mg} / \mathrm{kg}$ de $\mathrm{ZnSO} 4$ (Sanz-Fernandez et al., 2014); además, este mineral protege las células contra el daño oxidativo estabilizando las membranas, inhibiendo la enzima pro-oxidante nicotinamida adenina dinucleótido fosfato oxidasa (NADPH-Oxidasa), e induciendo la síntesis de metalotioneínas (Marreiro et al., 2017). Al respecto se ha informado que la suplementación con zinc induce la expresión de metalotioneínas en células Caco-2 (Wang et al., 2013), que podrían actuar como antioxidantes, debido a su capacidad para secuestrar especies reactivas de oxígeno e intermediarios de nitrógeno (Waeytens et al., 2009). Además, el zinc aumenta la expresión y la concentración de sustancias antimicrobianas como las $\beta$-defensinas en las células IPEC-J2 (Mao et al., 2013).

Por otro lado, el $\mathrm{Zn}$ es un componente estructural de la enzima superóxido dismutasa (SOD), presente en el citoplasma de las células; la SOD promueve la conversión de dos radicales superóxido $\left(\mathrm{O}_{2}^{-}\right)$en peróxido de hidrógeno $\left(\mathrm{H}_{2} \mathrm{O}_{2}\right)$ y oxígeno molecular $\left(\mathrm{O}_{2}\right)$, reduciendo la toxicidad de las especies reactivas de oxígeno (EROs); porque convierte una especie altamente reactiva en una menos dañina (Cruz y Soares, 2011; Marreiro et al., 2017). Los estudios también han destacado su papel en la regulación de la glutatión peroxidasa y en la expresión de la glutamato-cisteína ligasa, una enzima involucrada en la síntesis de glutatión que actúa directamente sobre la neutralización de radicales libres (Marreiro et al., 2017). En consecuencia, parece haber una variedad de mecanismos por los cuales el zinc en la dieta puede mantener o restaurar la integridad y morfología del epitelio intestinal, bajo condiciones de estrés oxidativo.

\section{CONCLUSIÓN}

La suplementación con $100 \mathrm{mg}$ de zinc/kg de alimento, a partir de metionina de zinc, mejora la integridad del epitelio intestinal en los cerdos de engorda, criados bajo condiciones de alta carga calórica, y la suplementación durante el periodo de gestaciónlactación, se puede utilizar como una estrategia para reducir la mortalidad durante la etapa de engorda. 


\section{LITERATURA CITADA}

AL MASRI S, Hünigen H, Al Aiyan A, Rieger J, Zentek J, Richardson K, Plendl J. 2015. Influence of age at weaning and feeding regimes on the postnatal morphology of the porcine small intestine. Journal Swine Health and Production. 23(4):186-203. ISSN: 1537-209X. http://dx.doi.org/10.13140/RG.2.1.1806.5043

BOUWHUIS MA, Sweeney T, Mukhopadhya A, Thornton K, McAlpine PO and O'Doherty JV. 2016. Zinc methionine and laminarin have growth-enhancing properties in newly weaned pigs influencing both intestinal health and diarrhoea occurrence. Journal of Animal Physiology and Animal Nutrition. 101(6):1273-1285. ISSN: 0931-2439. http://dx.doi.org/10.1111/jpn.12647

CHAND N, Naz S, Khan A, Khan S, Khan RU. 2014. Performance traits and immune response of broiler chicks treated with zinc and ascorbic acid supplementation during cyclic heat stress. International Journal of Biometeorology. 58(10):2153-2157. ISSN: 1432-1254. http://dx.doi.org/10.1007/s00484-014-0815-7

CHASAPIS CT, Loutsidou AC, Spiliopoulou CA, Stefanidou ME. 2012. Zinc and human health: an update. Archives of Toxicology. 86(4):521-534. ISSN: 0340-5761. http://dx.doi.org/10.1007/s00204-011-0775-1

CIAD. Centro de Investigación en Alimentación y Desarrollo A.C., Unidad Culiacán. Sistema Estadístico del Clima Automatizado de Sinaloa. 2015. http://187.141.135.166/CIAD/DatosPorPeriodoNuevo.aspx

CIAD. Centro de Investigación en Alimentación y Desarrollo A.C., Unidad Culiacán. Sistema Estadístico del Clima Automatizado de Sinaloa. 2016. http://187.141.135.166/CIAD/DatosPorPeriodoNuevo.aspx

CRUZ JBF, Soares HF. 2011. Uma revisão sobre o zinco. Ensaios Ciência Ciências Biológicas Agrárias Saúde. 15: 207-222. ISSN: 1415-6938. https://www.redalyc.org/pdf/260/26019329014.pdf

ESHEL GM, Safar P, Stezoski W. 2001. The role of thegut in the pathogenesis of death due to hyperthermia. The American Journal of Forensic Medicine and Pathology. 22(1):100-104. ISSN: 0195-7910. http://dx.doi.org/10.1097/00000433-200103000-00022 
LAGANA C, Ribeiro AML, Kessler A, Kratz LR, Pinheiro CC. 2007. Effect of the supplementation of vitamins and organic minerals on the performance of broilers under heat stress. Revista Brasileira de Ciencia Avícola. 9(1):01-06. ISSN: 1806-9061. http://dx.doi.org/10.1590/S1516-635X2007000100006

LAMBERT GP, Gisolfi CV, Berg DJ, Moseley PL, Oberley LW, Kregel KC. 2002. Selected contribution: Hyperthermia-induced intestinal permeability and the role of oxidative and nitrosative stress. Journal Applied of Physiology. 92(4):1750-1761. ISSN: 1522-1601. http://dx.doi.org/10.1152/japplphysiol.00787.2001

LAMBERT GP. 2009. Stress-induced gastrointestinal barrier dysfunction and its inflammatory effects. Journal of Animal Science. 87:E101-E108. ISSN: 1525-3163. http://dx.doi.org/10.2527/jas.2008-1339

LI BT, van Kessel AG, Caine WR, Huang SX, Kirkwood RN. 2001. Small intestinal morphology and bacterial populations in ileal digesta and feces of newly weaned pigs receiving a high dietary level of zinc oxide. Canadian Journal of Animal Science. 81(4):511-516. ISSN: 0008-3984. https://doi.org/10.4141/A01-043

LI Y, Cao Y, Zhou X, Wang F, Shan T, Li Z, Xu W, Li C. 2015. Effects of zinc sulfate pretreatment on heat tolerance of Bama miniature pig under high ambient temperature. Journal of Animal Science. 93:3421-3430. ISSN: 1525-3163. http://dx.doi.org/10.2527/jas.2015-8910

LONG L, Chen J, Zhang Y, Liang X, Ni H, Zhang B, Yin Y. 2017. Comparison of porous and nano zinc oxide for replacing high-dose dietary regular zinc oxide in weaning piglets. Plos ONE. 12(8):e0182550. ISSN: 1932-6203. https://doi.org/10.1371/journal.pone.0182550

MADER TL, Davis MS, Brown-Brandl T. 2006. Environmental factors influencing heat stress in feedlot cattle. Journal of Animal Science. 84:712-719. ISSN: 0021-8812. http://digitalcommons.unl.edu/cgi/viewcontent.cgi?article=1622\&context=animalscifacpu b

MANI V, Weber TE, Baumgard LH, Gabler NK. 2012. Growth and development symposium: Endotoxin, inflammation, and intestinal function in livestock. Journal of animal Science. 90 (5):1452-1465. ISSN: 0021-8812. http://dx.doi.org/10.2527/jas.2011-4627

MAO X, Qi S, Yu B, He J, Yu J, Chen D. 2013. Zn (2+) and L-isoleucine induce the expressions of porcine beta-defensins in IPEC-J2 cells. Molecular Biology Reports. 40(2): 1547-1552. ISSN: 1573-4978. http://dx.doi.org/10.1007/s11033-012-2200-0 
MARET W. 2013. Zinc biochemistry: From a single zinc enzyme to a key element of life. Advances in Nutrition. 4(1):82-91. ISSN: 2161-8313. http://dx.doi.org/10.3945/an.112.003038.

MARREIRO DN, Cruz KJC, Morais JBS, Beserra JB, Severo, de Oliveira ARS. 2017. Zinc and Oxidative Stress: Current Mechanisms. Antioxidants, 6 (2): 24. ISSN: 2076-3921. http://dx.doi.org/10.3390/antiox6020024

McDOWELL LR. 2003. Zinc. En Minerals in Animal and Human Nutrition. 2nd ed. Elsevier, Amsterdam, The Netherlands. Pp. 644. ISBN: 978-0-444-51367-0; https://www.sciencedirect.com/book/9780444513670/minerals-in-animal-and-humannutrition

MING-ZHE L, Jie-Ting H, Yi-Hao T, Syuan-Yian M, Chao-Ming F, Tu-Fa L. 2016. Nanosize of zinc oxide and the effects on zinc digestibility, growth performances, immune response and serum parameters of weanling piglets. Animal Science Journal. 87: 13791385. ISSN: 1740-0929. http://dx.doi.org/10.1111/asj.12579

NOM-033. 2014. NOM-033-SAG. ZOO-2014. Métodos para dar muerte a los animales domésticos y silvestres, 26 . https://www.gob.mx/profepa/documentos/norma-oficialmexicana-nom-033-sag-zoo-2014-metodos-para-dar-muerte-a-los-animalesdomesticos-y-silvestres

NRC (National Research Council). 2012. Nutrient requirements of swine. 11th rev. ed. Natl. Acad. Press, Washington, DC. USA.Pp. 420. ISBN: 978-0-309-22423-9; https://www.nap.edu/catalog/13298/nutrient-requirements-of-swine-eleventh-revisededition

PAYNE RL, Bidner TD, Fakler TM and LL Southern. 2006. Growth and intestinal morphology of pigs from sows fed two zinc sources during gestation and lactation. Journal of Animal Science. 84:2141-214. ISSN: 0021-8812, https://dx.doi.org/10.2527/jas.2005627

PEARCE SC, Gabler NK, Ross JW, Escobar J, Patience JF, Rhoads RP, Baumgard LH. 2013a. The effects of heat stress and plane of nutrition on metabolism in growing pigs. Journal of Animal Science. 91:2108-2118. ISSN: 1525-3163. http://dx.doi.org/10.2527/jas.2012-5738 
PEARCE SC, Mani V, Boddicker RL, Johnson JS, Weber TE, Ross JW, Rhoads RP, Baumgard LH, Gabler NK. 2013b. Heat stress reduces intestinal barrier integrity and favors intestinal glucose transport in growing pigs. PLOS ONE. 8:E70215. ISSN: 19326203. http://dx.doi.org/10.1371/journal.pone.0070215

PEARCE SC, Mani V, Weber TE, Rhoads RP, Patience JF, Baumgard LH, Gabler NK. 2013c. Heat stress and reduced plane of nutrition decreases intestinal integrity and function in pigs. Journal of Animal Science. 91:5183-5193. ISSN: 1525-3163. http://dx.doi.org/10.2527/jas.2013-6759

PEARCE SC, Sanz-Fernandez MV, Torrison J, Wilson ME, Baumgard LH, Gabler NK. 2015. Dietary organic zinc attenuates heat stress-induced changes in pig intestinal integrity and metabolism. Journal of Animal Science. 93:4702-4713. ISSN: 1525-3163. http://dx.doi.org/10.2527/jas2015-9018

PEI X, Xiao Z, Liu L, Wang G, Tao W, Wanga M, Zou J, Leng D. 2018. Effects of Dietary Zinc Oxide Nanoparticles Supplementation on Growth Performance, Zinc Status, Intestinal Morphology, Microflora Population, and Immune Response in Weaned Pigs Running. Journal of the Science of Food and Agriculture. 99(3):1366-1374. ISSN: 10970010. http://dx.doi.org/10.1002/jsfa.9312

PROPHET E, Mills B, Arrington J, Sobón L. 1995. Métodos histotecnológicos. Instituto de Patología de las Fuerzas Armadas de los Estados Unidos de América. Washington DC. Registro de Patología de los Estados Unidos de América (ARP) e Instituto de Patología de las Fuerzas Armadas de los Estados Unidos de América (AFIP). ISBN: 1881041212 9781881041214; histotecnologicos/oclc/630264753 https://www.worldcat.org/title/metodos-

RAKHSHANDEH A, Dekkers JCM, Kerr BJ, Weber TE, English J, Gabler NK. 2012. Effect of immune system stimulation and divergent selection for residual feed intake on digestive capacity of the small intestine in growing pigs. Journal of animal Science. 90(Suppl. 4):233-235. ISSN: 0021-8812. http://dx.doi.org/10.2527/jas.53976

RENAUDEAU D, Gourdine JL, St-Pierre NR. 2011. A meta-analysis of the effects of high ambient temperature on growth performance of growing finishing pigs. Journal of Animal Science. 89(7):2220-2230. SSN: 1525-3163. http://dx.doi.org/10.2527/jas.2010-3329

RICHARDS JD, Fisher PM, Evans JL, Wedekind KJ. 2015. Greater bioavailability of chelated compared with inorganic zinc in broiler chicks in the presence or absence of elevated calcium and phosphorus. Open Access Animal Physiology. 7:97-109. ISSN: 1179-2779. https://doi.org/10.2147/OAAP.S83845 
ROMO JM, Romo JA, Barajas R, Enríquez I, Silva G, Montero A. 2017. Efecto del consume de zinc orgánico en la respuesta productiva de la cerda y su camada. Abanico veterinario. 7(2):43-59. ISSN 2448-6132. http://dx.doi.org/10.21929/abavet2017.72.4

SANZ-FERNANDEZ MV, Pearce SC, Gabler NK, Patience JF, Wilson ME, Socha MT, Torrison JL, Rhoads RP, Baumgard LH. 2014. Effects of supplemental zinc amino acid complex on gut integrity in heat-stressed growing pigs. Animal. 8:43-50. ISSN: 1751732X. http://dx.doi.org/10.1017/S1751731113001961

SCHLEGEL P, Sauvant D, Jondreville C. 2013. Bioavailabiliy of zinc sources and their interaction with phytates in broilers and piglets. Animal. 7(1):47-59. ISSN: 1751-732X. http://dx.doi.org/10.1017/S1751731112001000

STEEL GD y Torrie JH. 1985. Bioestadística: Principios y Procedimientos. (2da. Ed.) McGraw-Hill, México, DF. Pp. 624. ISBN: 968-451495-6; https://www.academia.edu/35066774/Steel_Robert_G__Bioestadistica_Principios_Y_Procedimientos_2ed

ST-PIERRE NR, Cobanov B, Schnitkey G. 2003. Economic losses from heat stress by US livestock industries. Journal of Dairy Science. 86(Suppl):E52-E77. ISSN: 0022-0302. https://doi.org/10.3168/jds.S0022-0302(03)74040-5

TANG M, Laarveld B, Van Kessel AG, Hamilton DL, Estrada A, Patience JF. 1999. Effect of segregated early weaning on postweaning small intestinal devel-opment in pigs. Journal of Animal Science. 77(12):3191-3200. ISSN: 1525-3163. https://doi.org/10.2527/1999.77123191x

WAEYTENS A, De Vos M, Laukens D. 2009. Evidence for a Potential Role of Metallothioneins in Inflammatory Bowel Diseases. Mediators of Inflammation. Article ID 729172: 9 pages. ISSN: 0962-9351. http://dx.doi.org/10.1155/2009/729172

WANG X, Valenzano MC, Mercado JM, Zurbach EP, Mullin JM. 2013. Zinc supplementation modifies tight junctions and alters barrier function of CACO-2 human intestinal epithelial layers. Digestive Diseases and Sciences. 58(1): 77-87. ISSN: 01632116. http://dx.doi.org/10.1007/s10620-012-2328-8

ZHANG B, Guo Y. 2009. Supplemental zinc reduced intestinal permeability by enhancing occludin and zonula occludens protein-1 (ZO-1) expression in weaning piglets. The British Journal of Nutrition. 102:687-693. ISSN: 1475-2662. http://dx.doi.org/10.1017/S0007114509289033 